\title{
Association between ovarian endometriosis and malignancy in the peri-menopausal period: report of two cases and review of the literature
}

\author{
Eugenio Volpi • Elisa Peano • Annamaria Ferrero • \\ Luciano Mosso • Alberto Daniele • Piero Sismondi
}

Received: 6 June 2008 / Accepted: 25 August 2008 / Published online: 25 September 2008

(C) Springer-Verlag 2008

\begin{abstract}
The association of endometriosis and ovarian malignancy is about $1 \%$, with a peak incidence in patients over 45 years. The best way of treating peri- and postmenopausal women with endometriosis is still a very controversial issue. We report two cases of peri-menopausal women in which endometriosis was associated to ovarian malignancy. The first case was a 49-year-old woman who underwent laparoscopic oophorectomy for an ovarian endometrioid cyst. Definitive histology showed ovarian endometrioid cyst with endometrioid and clear cell carcinoma. Subsequently, surgical restaging was performed. The other case was a 49-year-old woman who underwent hysterectomy and bilateral oophorectomy for an ovarian mass suggestive of endometriosis and uterine myomas. At frozen section examination, ovarian adenocarcinoma was found and staging procedure was performed. Definitive histology showed carcinosarcoma of the ovary with areas of endometriod adenocarcinoma. Given the non-infrequent association between ovarian endometriosis and cancer, more data are needed to decide for an aggressive or conservative approach to perimenopausal endometriosis.
\end{abstract}

E. Volpi $\cdot$ E. Peano $\cdot$ A. Ferrero $(\bowtie) \cdot$ L. Mosso $\cdot$ A. Daniele $\cdot$

P. Sismondi

Academic Division of Gynaecological Oncology,

Institute for Cancer Research and Treatment (IRCC) of Candiolo

\& AO Ordine Mauriziano,

Largo Turati, 62-

10128 Turin, Italy

e-mail: a.ferrero@katamail.com

L. Mosso

Department of Pathology, AO Ordine Mauriziano,

Turin, Italy
Keywords Cystic endometriosis · Ovarian cancer . Carcinosarcoma $\cdot$ Peri-menopausal period

\section{Introduction}

Endometriosis is the presence of endometrial-like tissue outside the uterus, which induces a chronic inflammatory reaction. It is a common health problem among women with a prevalence around $6-10 \%$ in the general female population [1]; in women with pelvic pain, dyspareunia, dysmenorrhea, or subfertility, the frequency is $35-50 \%$ $[2,3]$.

The clinical presentation of endometriosis is variable among patients and often overlaps with other conditions such as irritable bowel syndrome and pelvic inflammatory disease. However, a consistent number of women are asymptomatic.

Transvaginal sonography (TV US) plays an important role in the detection of cystic endometriosis [4-6]. Sensitivity of TV US is about $92 \%$ and specificity is about $96 \%$ [7].

Magnetic resonance imaging (MRI) has emerged as the best imaging modality to diagnose and evaluate the extent of deep pelvic endometriosis in a specific location, such as the uterosacral ligaments, the bladder, the vagina, the rectovaginal sectum, and the rectosigmoid, and shows high accuracy in cystic endometriosis $[8,9]$. Nevertheless, some authors do not recommend MRI as a first-line exam because of its limited diagnostic value compared to laparoscopy [10], and the gold standard for the diagnosis of endometriosis is still laparoscopy and histology.

We do not routinely perform pelvic MRI for ovarian endometriosis before surgery. The term peri-menopause is defined as about or around menopause and the transition from the reproductive phase to the non-reproductive state. It 
begins with variation in menstrual cycle length in a woman who has monotropic FSH rise and ends with the final menstrual period (not able to be recognized until after 12 months of amenorrhea). The menopausal transition usually begins in a woman's 40s [11].

Reported data show that the association of ovarian malignancy and endometriosis is about $1 \%$ with a peak incidence in patients over 45 years $[12,13]$. The mechanism through which endometriosis may be associated with ovarian cancer is unclear. One possibility is that both share similar risk factors, for instance nulliparity, late age at birth of a first child, family history of ovarian cancer, Caucasian race, early menarche, and late menopause, instead tubal ligation, hysterectomy, oral contraception, and breast feeding decrease this risk. The best way of treating periand post-menopausal women with endometriosis is still a very controversial issue.

From 2001 to 2007, 155 patients underwent laparoscopy for ovarian endometriosis in our Institution. Fifty-two $(33.5 \%)$ of those women were $40-50$ years old with a mean age of 46 years.

Minimal and mild endometriosis are excluded from this series since all our patients were treated for ovarian cyst. In the same period of time, 147 ovarian cancer cases were treated in our institution. Two cases $(1.29 \%)$ in which endometriosis was associated to ovarian malignancy were identified. Both patients were in the peri-menopausal period. We report about these two cases.

\section{Case 1}

In May 2006, a 49-year-old woman presented with light pelvic pain and irregular vaginal bleeding for the last 3 months. Parity was two, the age at time of first pregnancy was 28 , her breastfeeding lasted 13 months in total, menses were regular, she never had infertility, and oral contraceptives were used for 1 year. No previous ovarian cancer cases were found in her family.

In 2001, she had a laparoscopic oophorectomy on the left side because of an endometriotic cyst. At pelvic examination, a right ovarian mass was found. TV US showed normal uterus and a right ovarian cyst of hypoechoic echogenicity $(9 \times 6 \mathrm{~cm})$. Ca 125 was negative. Endometriotic cyst was suspected according to the sonographic criteria reported in the literature [14].

Oophorectomy was performed laparoscopically. During the mobilization of the ovary, spillage of the cystic content happened. Definitive histology showed microscopic endometrioid adenocarcinoma with foci of solid and clear cell carcinoma, grade 3, within an ovarian endometrioid cyst. One month later, she underwent a laparoscopic restaging: peritoneal washing, hysterectomy, omentectomy, and random biopsies with para-aortic, infra, and sovra mesenteric and pelvic lymph nodes sampling were performed. Histology was negative in all the samples, including 15 para-aortic lymph nodes and 11 ipsilateral pelvic lymph nodes. Finale stage was pT1c because of the spillage. Even if the chemotherapeutic management of pT1c and third stage ovarian cancer is the same, we considered it important to have data about cytology in the peritoneum after spillage and about the lymph nodes. The patient received six cycles of carboplatin-Taxol combination as adjuvant chemotherapy. At 2 years follow-up, she was well with no evidence of disease.

\section{Case 2}

In June 2006, a 49-year-old woman presented with a pelvic mass. In addition, she complained of urge incontinence.

Parity was 1 (in 1984), she used oral contraceptive pill for 7 months, no sterility medications were adopted, and her family history was negative for malignancy. In 2000, she had a sonographic diagnosis of endometriosis and myomas of the uterus.

TV US showed a uterus of increased volume because of myomas. Endometrium was $7.6 \mathrm{~mm}$ thick. The left ovary had an hypoechoic homogeneous cyst $(54 \times 45 \mathrm{~mm})$ suggesting endometriosis, and the right ovary had similar features measuring $102 \times 100 \mathrm{~mm}$. No ascites was present in the Douglas pouch. The same features were present in the previous ultrasonographic controls. RMN confirmed the same findings.

Up to September 2006, she refused the surgical procedure and eventually she underwent surgery in November 2006. At the first referral in June, pelvic examination showed a pelvic mass comparable to a 13week pregnancy. However, at the hospital admission in November, an important increase of the mass reaching the transverse umbilical line was found. Ca 125 in serum was $549 \mathrm{UI} / 1$ in June and decreased to $205 \mathrm{UI} / 1$ in September. The woman was advised to have hysterectomy and bilateral oophorectomy via laparotomy because the abdominal-pelvic mass was fixed at bimanual examination. The diagnosis at frozen section examination of the left ovary was adenocarcinoma, thus a staging surgical procedure was performed.

Definitive histology showed carcinosarcoma of the ovary with areas of endometriod adenocarcinoma, grade 3 (Fig. 1). Some areas of endometriosis and atypical hyperplasia were found close to the tumor (Fig. 2). Thirty-eight lymph nodes were collected, and at histological examination, they were free from malignant cells. Final stage was pT2aN0. The patient underwent chemotherapy with ifosfamide and cisplatin combination. The patient was disease free at 25 months follow-up. 


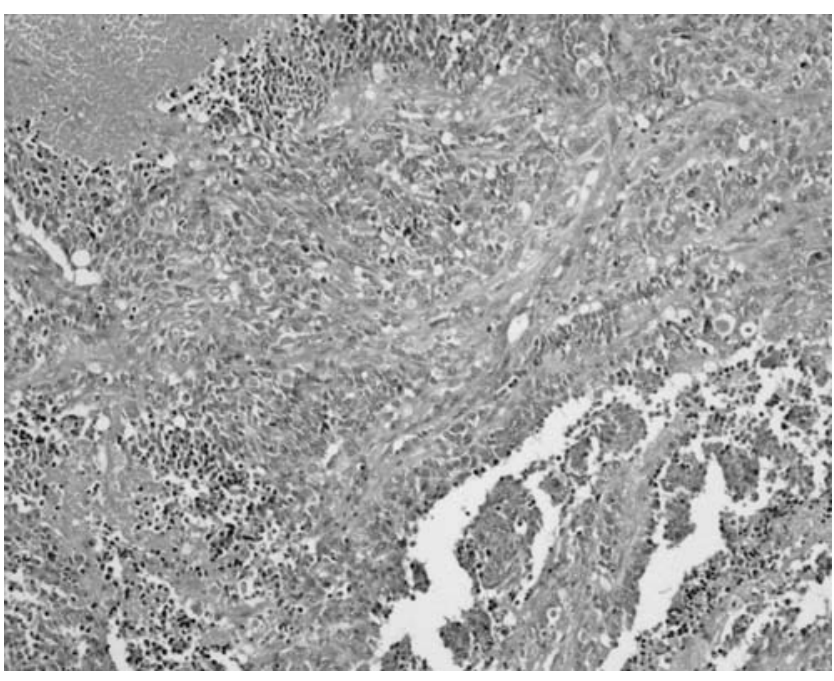

Fig. 1 Foci of adenocarcinoma with sarcomatous component and areas of necrosis

\section{Discussion}

In 1925, Sampson first proposed the criteria still in use to identify malignant tumors raised from endometriosis: (1) clear evidence of endometriosis close to the tumor, (2) the cancer must arise in endometriosis and not invade it from another site, and (3) it must be possible to recognize the characteristic histological structure of endometriosis with both glands and surrounding stroma [15]. In 1953, Scott added an even stricter criterion of a histologically proven transition from benign endometriosis to cancer [16]. An association of cancer and endometriosis is evident even if no causal relationship can be proven. The frequency of malignant transformation of endometriosis is unknown, but it is estimated that $1 \%$ or less of women with endometriosis will develop endometriosis-associated neoplasms [17].

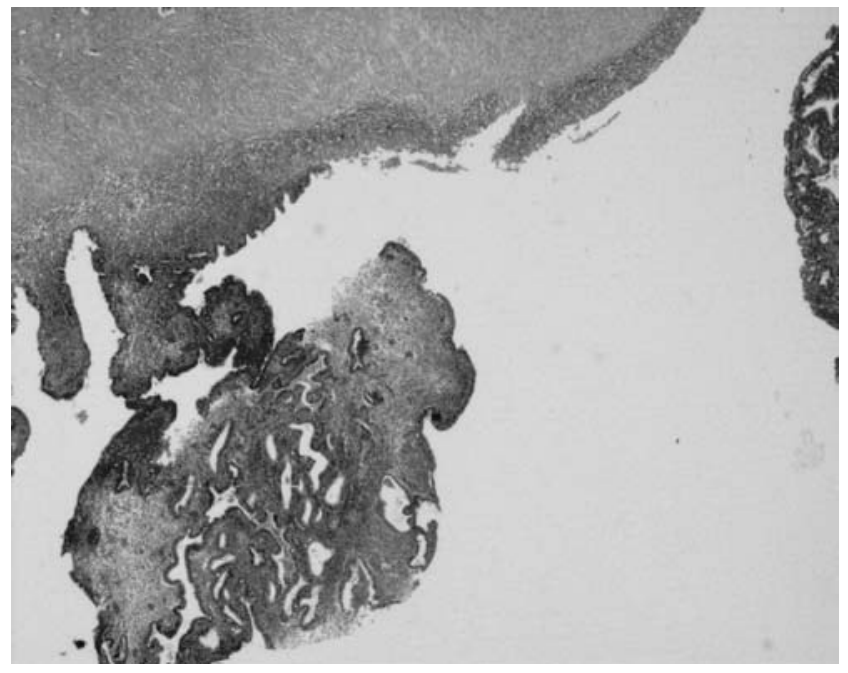

Fig. 2 Cystic endometriosis and areas of atypical hyperplasia
Some authors suggest that this association may be more frequent than reported [18].

Endometriosis was found to be concomitantly present to ovarian tumors in $4-29 \%$ of cases operated for malignancy [19]. Most represented histotypes are clear cell carcinoma and endometrioid adenocarcinoma (about 60-70\%) [20, 21]. The clear association between endometriosis and endometrioid and/or clear cell carcinomas supports a possible casual relationship between endometriosis and ovarian cancer [19].

The combination of these two histotypes was found in our first patient. Carcinosarcoma, which we found in our second patient, is considered a rare variety. Both tumors are very aggressive histotypes. Nevertheless, it has been reported that endometriosis-associated ovarian cancer has a better prognosis than the non-associated one [19]. Despite that we cannot draw any conclusion, our patients are still well till now.

Data from the majority of the available cohort and casecontrol studies on patients with endometriosis tend to suggest an association between endometriosis and ovarian cancer $[19,21]$ (Table 1).

Ness et al. pooled eight case-control studies to evaluate the influence of infertility on ovarian cancer: endometriosis was linked with a significantly increased risk (odd ratio 1.7, CI 1.1-2.7) [22]. Brinton et al. performed the largest cohort study investigating the risk of cancer after hospitalization for endometriosis in Sweden: a significant risk of increase for ovarian cancer was observed with a standardized incidence ratio (SIR) of 1.9 [23].

The simultaneous finding of ovarian endometriosis and cancer increases with the age of the patients $[12,13]$. In a cohort study evaluating the risk of developing ovarian cancer among women with ovarian endometrioma, the excess risk for ovarian cancer increased markedly with increasing age at ovarian endometrioma diagnosis, from $\mathrm{SIR}=3.88$ in women younger than 30 years to $\mathrm{SIR}=13.2$ in those 50 years or older $(P=0.014)$ [24]. Moreover, recent data suggest that women with early diagnosed and long-standing endometriosis have a higher risk of developing ovarian cancer [25].

In our experience, two patients in peri-menopausal age had ovarian endometriosis associated to malignancy. The

Table 1 Endometriosis and ovarian cancer risk in clinical and epidemiological studies (RR, relative risk, 95\% CI confidence interval; numbers in square brackets are reference citations)

\begin{tabular}{llcc}
\hline Case control/cohort study & \multirow{2}{*}{ Size cohort } & \multicolumn{2}{c}{ Ovarian cancer } \\
\cline { 3 - 4 } & & RR & $95 \%$ CI \\
\hline Briton et al. [23] & 20,686 & 1.92 & $1.3-2.8$ \\
Ness et al. [22] & 3,678 cases/5,268 & 1.73 & $1.10-2.71$ \\
& $\quad$ controls & & \\
Melin et al. [25] & 64,492 & 1.43 & $1.19-1.71$ \\
Kobayashi et al. [24] & 6,398 & 8.95 & $4.12-15.3$ \\
\hline
\end{tabular}


first patient was already operated for endometriosis, and even at the second surgical approach, the macroscopic impression was consistent. Only definitive histology diagnosed adenocarcinoma. In the second case, the patient had been followed up for a long time and she became symptomatic only few months before surgery. The sarcoma histotype could justify this clinical presentation.

TV US has changed the approach to diagnosis of cystic endometriosis, and most of the procedures are started laparoscopically because of the accuracy of TV US. We usually treat endometriosis by laparoscopy. Our second patient underwent laparotomy because of the great increase of the pelvic mass. Both patients had no other important risk factors for ovarian cancer.

Serum level of Ca 125 is a marker of ovarian cancer. The role of $\mathrm{Ca} 125$ in endometriosis is discussed, especially in peri-menopausal patients. Actually, in case of endometriosis, the blood value of $\mathrm{Ca} 125$ is expected to increase $[10,26]$. In our second case, we registered a severe reduction of the level of $\mathrm{Ca} 125$ (about 50\% less), and this has acted as a confounding element.

Guidelines do not specify an elective treatment for perimenopausal women affected by endometriosis of the ovary. Due to the natural involution of the endometriod disease during the postmenopausal period [3], the most frequent attitude could be a non-surgical approach. Nevertheless, in the age ranging between 45 and 55 years, the incidence of ovarian cancer is relevant (about 10-20/100,000) and despite the widely spread introduction of new antineoplastic agents, the overall 5-year survival rate remains 30\% [27].

Obviously, it is mandatory to outweigh risks and benefits of removing a woman's ovaries in the peri-menopausal stage. Hormonal replacement therapy can be a valid support when estrogen deficiency is caused by removing ovaries; indeed, each patient has to be evaluated singularly about the feasibility of HRT, but it looks safe enough [28].

Some authors support the findings that ovarian endometriosis is not an innocuous lesion even in perimenopausal patients and suggest that complete excision of endometriosis should be attempted if fertility is not desired in these patients [21].

Other authors do not support that "prophylactic surgery" should be offered systematically in women with endometriosis, while oral contraception, a demonstrated strong protective factor of ovarian cancer, should be favored [19].

Given the non-infrequent association between ovarian endometriosis and cancer, we think the issue of an aggressive or conservative approach to peri-menopausal endometriosis should be discussed.

\section{References}

1. Vigano P, Parazzini F, Somigliana E et al (2004) Endometriosis: epidemiology and aetiological factors. Baillieres Best Pract Res Clin Obstet Gynaecol 18:177-200

2. Giudice LC, Kao LC (2004) Endometriosis. Lancet 364:1789-1799

3. Cramer DW, Missmer SA (2002) The epidemiology of endometriosis. Ann N Y Acad Sci 955:11-22

4. Volpi E, De Grandis T, Zuccaro G et al (1995) Role of transvaginal sonography in the detection of endometriomata. J Clin Ultrasound 23:163-167

5. Bhatt S, Kocakoc E, Dogra VS (2006) Endometriosis: sonographic spectrum. Ultrasound Q 22:273-280

6. Kurjak A, Kupesic S (1995) Scoring system for prediction of ovarian endometriosis based on transvaginal color and pulsed Doppler sonography. Fertil Steril 63:1136-1137

7. Pascual MA, Tresserra F, Lopez-Marin L et al (2000) Role of color Doppler ultrasonography in the diagnosis of endometriotic cyst. J Ultrasound Med 19:695-699

8. Bazot M, Darai E, Hourani R (2004) Deep pelvic endometriosis: MR imaging for diagnosis and prediction of extension of disease. Radiology 232:379-89

9. Bazot M, Darai E (2005) Sonography and MR imaging for the assessment of deep pelvic endometriosis. J Minim Invasive Gynecol 12:178-185

10. Kennedy S, Bergqvist A, Chapron C et al (2005) ESHRE guidelines for endometriosis and treatment of endometriosis. Hum Reprod 20:2698-2704

11. North American Menopause Society (2004) Treatment of menopause-associated vasomotors symptoms: position statement of The North American Menopause Society. Menopause 11(1):11-33

12. Sainz de la Cuesta R, Eichorn JH et al (1996) Histologic transformation of benign endometriosis to early epithelial ovarian cancer. Gynecol Oncol 60:238-244

13. De Priest PD, Banks ER, Powell DE et al (1992) Endometriod carcinoma of the ovary and endometriosis: the association in postmenopausal women. Gynecol Oncol 47:71-75

14. Van Calster B, Timmerman D, Bourne T (2007) Discrimination between benign and malignant adnexal masses by specialist ultrasound examination versus serum Ca-125. J Natl Cancer Inst 99:1706-1714

15. Sampson JA (1925) Endometrial carcinoma of ovary arising in endometrial tissue in that organ. Arch Surg 10:1-72

16. Scott RB (1953) Malignant changes in endometriosis. Obstet Gynecol 2:283-289

17. Heaps JM, Nieberg RK, Berek JS (1990) Malignant neoplasms arising in endometriosis. Obstet Gynecol 75:1023-1028

18. Stern RC, Dash R, Bentley RC et al (2001) Malignancy in endometriosis: frequency and comparison of ovarian and extraovarian types. Int J Gynecol Pathol 20:133-139

19. Somigliana E, Viganò P, Parazzini F et al (2006) Association between endometriosis and cancer: a comprehensive review and a critical analysis of clinical and epidemiological evidence. Gynecol Oncol 101:331-341

20. Steed H, Chapman W, Laframboise S (2004) Endometriosisassociated ovarian cancer: a clinicolpathologic review. J Obstet Gynaecol Can 26:709-715

21. Van Gorp T, Amant F, Neven P (2004) Endometriosis and the development of malignant tumor of the pelvis: a review of 
literature. Baillieres Best Pract Res Clin Obstet Gynaecol 18:349-371

22. Ness RB, Cramer DW, Goodman MT et al (2002) Infertility, fertility drugs, and ovarian cancer: a pooled analysis of casecontrol studies. Am J Epidemiol 155:217-224

23. Brinton LA, Gridley G, Persson I et al (1997) Cancer risk after a hospital discharge diagnosis of endometriosis. Am J Obstet Gynecol 176:572-579

24. Kobayashi H, Sumomoto K, Moniwa N et al (2007) Risk of developing ovarian cancer among women with ovarian endometrioma: a cohort study in Shizuoka, Japan. Int J Gynecol Cancer 17:37-43
25. Melin A, Sparén P, Persson I et al (2006) Endometriosis and the risk of cancer with special emphasis on ovarian cancer. Hum Reprod 21:1237-1242

26. Royal College of Obstetricians and Gynaecologists (2004) RCOG guidelines no. 24, October 2006

27. Jemal A, Murray T, Samuels A et al (2003) Cancer statistics 2003. CA Cancer J Clin 53:5-26

28. Matorras R, Elorriaga MA, Pijoan JI (2002) Recurrence of endometriosis in women with bilateral adnexectomy (with or without total hysterectomy) who received hormone replacement therapy. Fertil Steril 77(2):303-308 